\title{
EXCITATION OF THE CHANDLER WOBBLE \\ BY LARGE EARTHQUAKES
}

\author{
KUNIHIKO SHIMAZAKI \\ Earthquake Research Institute, Tokyo University, Japan \\ and \\ HITOSHI TAKEUCHI \\ Geophysical Institute, Faculty of Science, Tokyo University, Japan
}

\begin{abstract}
The direction of anomalous polar motion is compared with that of the motion expected from the source mechanism generating the earthquakes associated with the motion. The result supports the hypothesis that major earthquakes excite the Chandler wobble.
\end{abstract}

\section{Introduction}

The Chandler wobble may be considered to be a succession of free damped nutations of the Earth excited randomly in time. Recently the hypothesis has been presented that major earthquakes excite the Chandler wobble (Mansinha and Smylie, 1967; Ben-Menahem and Israel, 1970). This hypothesis is based on the recent development of the elasticity theory of dislocations in seismology. Smylie and Mansinha (1968) analysed the observed polar path and showed a significant correlation between the occurrence of specific seismic events and pole-path breaks of the BIH data.

To confirm this hypothesis it is needed to compare the specific anomalous polar motions with the focal mechanisms of the earthquakes associated with the motions. In the present study the directions of the anomalous motions are compared with those of motions expected from the source mechanisms of the earthquakes.

\section{Anomalous Polar Motions}

Based on Wiener's filtering theory, a 2-channel linear filter was designed to remove periodic motions from the polar motion. We denote by $m_{1}$ and $m_{2}$ the co-ordinates of the instantaneous pole, $m_{1}$ towards Greenwich and $m_{2}$ towards $90^{\circ} \mathrm{E}$ longitude. Prediction-errors $e_{1}$ and $e_{2}$ are defined as follows:

$$
\begin{aligned}
e_{1}(t) & =m_{1}(t)-n_{1}(t) \\
& =m_{1}(t)-\sum_{s=1}^{p}\left(f_{11}(s) f_{12}(s)\right)\left(m_{1}(t-s) m_{2}(t-s)\right)^{T} \\
e_{2}(t) & =m_{2}(t)-n_{2}(t) \\
& =m_{2}(t)-\sum_{s=1}^{p}\left(f_{21}(s) f_{22}(s)\right)\left(m_{1}(t-s) m_{2}(t-s)\right)^{T}
\end{aligned}
$$

$T=$ transposed matrix; where $n_{1}$ and $n_{2}$ are the predicted values of $m_{1}$ and $m_{2}$, 
respectively. The filter $f_{i j}$ is constructed using the condition that the time average of the squared sum of the prediction-errors should be a minimum.

In the problem under consideration the input functions are not Dirac functions but step functions, namely the abrupt changes in the products of inertia. Therefore the required anomalous polar motions are not revealed in the prediction-errors, but in the prediction-error change, which are defined as follows:

$$
\begin{aligned}
& d_{1}(t)=e_{1}(t)-e_{1}(t-1) \\
& d_{2}(t)=e_{2}(t)-e_{2}(t-1)
\end{aligned}
$$

Here we denote by $D(t)=\left(d_{1}(t)^{2}+d_{2}(t)^{2}\right)^{1 / 2}$, the magnitude of the prediction-error change.

The 2-channel linear filtering method was applied to the BIH pole-path for the period 1955.12.8-1969.6.27.

Figure 1 shows the co-ordinates of the BIH pole-path, $m_{1}$ and $m_{2}$; the predictionerrors, $e_{1}$ and $e_{2}$; and the magnitude of the prediction-error change $D$ for the period from 1957 to 1959 . The time of occurrence of large earthquakes with magnitude larger than 7.5 is denoted by the solid circle. It should be noticed that the solid circles coincide well with the peaks of the magnitude of the prediction-error change.

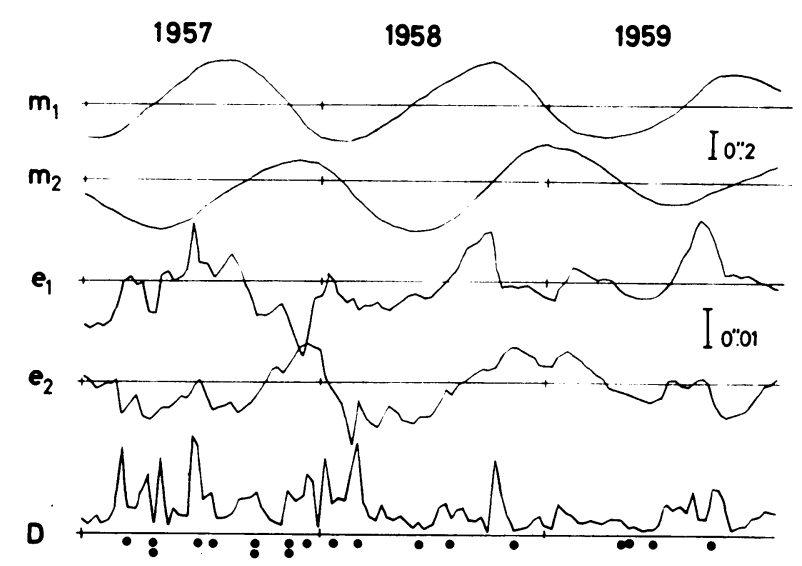

Fig. 1. The co-ordinates of the BIH pole-path, $m_{1}$ and $m_{2}$; the prediction-errors, $e_{1}$ and $e_{2}$; and the magnitude of the prediction-error change, $D$ for the period from 1957 to 1959.

\section{Apparent Precursory Phenomena of Large Earthquakes}

The average of the magnitude of the prediction-error change was calculated before and after the occurrence of the large earthquakes. It was shown to be statistically significant that the average is large 10 days before the occurrence of large earthquakes. Apparently precursory phenomena of large earthquakes were also reported by Smylie and Mansinha (1968). 
However, these apparently precursory phenomena can be attributed to the effect of the smoothing practice in the BIH data. Figure 2 shows the instantaneous pole position for each 10 days from March 4 to June 2 in 1968. The attached figures show the date. The upper part shows a slightly smoothed path obtained by averaging the unsmoothed BIH data for only 10 days. The lower part shows the smoothed one used

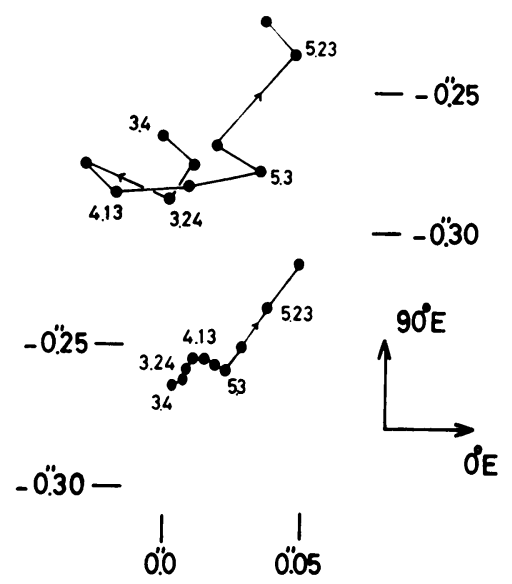

Fig. 2. A slightly smoothed pole-path and the smoothed BIH path for the period 1968.3.10 - 1968.6.2.

in the present study. On May 16 in 1968, the Tokachioki earthquake occurred in the northern part of Japan. Corresponding to this earthquake, the instantaneous pole position of May 23 is removed towards $90^{\circ} \mathrm{E}$ longitude. It should be noticed that only in the smoothed path is the pole position of May 13 also removed towards $90^{\circ} \mathrm{E}$ longitude. This, however, must be due to the smoothing. This kind of effect of smoothing may produce the apparent precursory phenomena of earthquakes.

\section{Directions of the Anomalous Polar Motions}

The directions of excitation of several earthquakes were computed from focal mechanisms of the earthquakes. The earthquake fault was represented by a point-source dislocation. The Earth was assumed to be a homogeneous, non-gravitating, elastic sphere. Density changes due to the displacements in the Earth were also neglected. These approximations lead to no significant error in the calculation of the direction of excitation because it is determined from the ratio of the changes in the inertia tensor elements.

Figure 3 shows the comparison of the direction of the anomalous polar motion with the direction expected from seismological observations. The solid squares show the cases in which both directions are obtained uniquely (the Ecuador earthquake of 1958, the Kuril Islands earthquake of 1963, the Alaska earthquake of 1964, the Tokachi- 
oki earthquake of 1968). The circles show the cases in which these directions are not obtained uniquely. The solid circle is the favourable one of the two solutions. The solid squares and the solid circles lie more or less on the line which indicated that the observed directions agree with the expected ones. These results are in agreement with the hypothesis that large earthquakes excite the Chandler wobble.

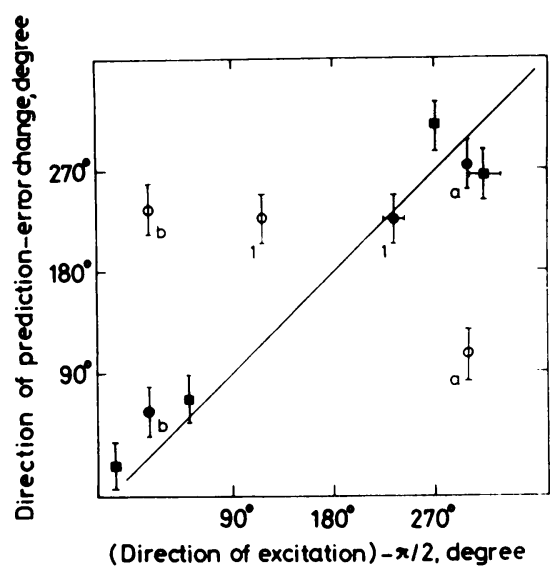

Fig. 3. The observed directions of the anomalous polar motion and the ones expected from earthquake mechanisms.

\section{References}

Balakina, L. M.: 1962, Izv. Ser. Geofiz. 1471.

Ben-Menahem, A. and Israel, M.: 1970, Geophys. J. 19, 367.

Kanamori, H.: 1970a, J. Geophys. Res. 75, 5011.

Kanamori, H.: 1970b, J. Geophys. Res. 75, 5029.

Mansinha, L. and Smylie, D. E.: 1967, J. Geophys. Res. 72, 4731.

Smylie, D. E., and Mansinha, L.: 1968, J. Geophys. Res. 73, 7661.

Stauder, W. and Bollinger, G. A.: 1966, Bull. Seismol. Soc. Am. 56, 1363.

\section{DISCUSSION}

Cheh Pan: The first error in the excitation of the Chandler wobble by large earthquakes was made by the first paper of Mansinha and Smylie, because their theoretical derivation is not really correct. According to their derivation, the dimensionless amplitude of the wobble is of the order of $10^{-14}$, if we use Munk and MacDonald's solution, it is $10^{-12}$. Actually it is $10^{-6}$; there is a discrepancy of $10^{-6}$ or $10^{-8}$. Secondly, the yearly rate of changes of the rotational product of inertia $I_{y z} \approx(C-B) \times$ $\times \dot{\theta} \sim 10^{33} \mathrm{~g} \mathrm{~cm}^{2} / \mathrm{yr}$, which is $10^{6}$ greater than what can be produced by earthquakes.

G.Pedersen: Previous commentator did believe that amplitudes of theoretical and observed wobble differ too drastically in Smylie and Mansinha's original paper. However the model has been much improved - first by Ben-Menahem and Israel to a spherical case and then by Smylie to a self gravitating elastic earth containing a liquid core.

Masanori Saito: In reply to Dr. Pan's comment; The point of this paper is that the observed direction of the pole agree quite well with ones predicted by earthquake mechanisms. The second point 
I wish to mention is that the focal dimensions as obtained by seismic waves seem to be consistently smaller than those obtained by geodetic measurements.

David E. Smith: I have a copy of Smylie and Mansinha's paper which has been accepted for publication by the Geophysical Journal in which the amplitude of the Chandler wobble is discussed and the relation to earthquakes. 